\title{
Placental mitochondrial DNA content is associated with childhood intelligence
}

\author{
Esmée M. Bijnens ${ }^{1,2^{*}} \mathbb{D}$, Catherine Derom ${ }^{2,3}$, Steven Weyers ${ }^{2}$, Bram G. Janssen ${ }^{1}$, Evert Thiery ${ }^{4}$ \\ and Tim S. Nawrot ${ }^{1,5}$
}

\begin{abstract}
Background: Developmental processes in the placenta and the fetal brain are shaped by the similar biological signals. Evidence accumulates that adaptive responses of the placenta may influence central nervous system development. We hypothesize that placental mtDNA content at birth is associated with intelligence in childhood. In addition, we investigate if intra-pair differences in mtDNA content are associated with intra-pair differences in intelligence.

Methods: Relative mtDNA content was measured using qPCR in placental tissue of 375 children of the East Flanders Prospective Twin Survey. Intelligence was assessed with the Wechsler Intelligence Scale for Children-Revised (WISC-R) between 8 and 15 years old. We accounted for sex, gestational age, birth weight, birth year, zygosity and chorionicity, cord insertion, age at measurement, indicators of socioeconomic status, smoking during pregnancy, and urban environment.

Results: In multivariable adjusted mixed modelling analysis, each doubling in placental mtDNA content was associated with 2.0 points ( $95 \% \mathrm{Cl} 0.02$ to $3.9 ; \mathrm{p}=0.05$ ) higher total and 2.3 points ( $95 \% \mathrm{Cl} 0.2$ to $4.3 ; \mathrm{p}=0.03$ ) higher performance IQ in childhood. We observed no association between mtDNA content and verbal intelligence. Intra-pair differences in mtDNA content and IQ were significantly $(p=0.01)$ correlated in monozygotic-monochorionic twin pairs, showing that the twin with the highest mtDNA content was 1.9 times more likely $(p=0.05)$ to have the highest IQ. This was not observed in dichorionic twin pairs.

Conclusions: We provide the first evidence that placental mtDNA content is associated with childhood intelligence. This emphasizes the importance of placental mitochondrial function during in utero life on fetal brain development with long-lasting consequences.
\end{abstract}

Keywords: Mitochondrial DNA content, Placenta, Intelligence, Childhood, Twins, DOHaD

\section{Background}

Studies suggest that the placenta, apart from transport of maternal nutrients, growth factors and hormones, also plays a pivotal role in central nervous development through adaptive responses to the maternal environment [1-3]. A suboptimal placental nutrient supply can influence the fine structure of neuronal networks in the brain [4], and therefore limiting final cognitive performance to a level below genetic potential [5]. Mitochondria are

\footnotetext{
*Correspondence: esmee.bijnens@uhasselt.be

${ }^{1}$ Centre for Environmental Sciences, Hasselt University, Agoralaan Building D, 3590 Diepenbeek, Belgium

Full list of author information is available at the end of the article
}

intracellular organelles and their main role is to generate energy for the cell. Since metabolic energy is important in the brain at the synaptic level for normal neural communication [6], mitochondria are essential for human cognition [7]. Therefore the maintenance of mitochondrial DNA (mtDNA) content is important to preserve mitochondrial function, cell growth and morphology [8]. Fetuses adapt their mitochondrial structure and metabolism when the supply of nutrients is limited [9] or due to exposure to environmental pollution [10].

It was demonstrated that human ageing entails a decline in mtDNA copy number (mtDNA content) among Danish twins and singletons (18-93 years of age) [11]. Moreover, subjects with low mtDNA copy 
number in blood had poorer outcomes in terms of physical strength, self-rated health, higher all-cause mortality and also cognitive performance, compared with subjects with high mtDNA copy number [11]. In elderly, a higher blood mtDNA copy number was consistently associated with higher cognitive performance [11, 12].

The Developmental Origins of Health and Disease (DOHaD) hypothesis, often called the Barker hypothesis, states that adverse influences of the early-life environment can result in permanent changes in adulthood [13]. In this regard, twin studies allow estimation of the importance of the individual fetoplacental environment unique to each fetus, while controlling for shared factors, such as maternal environment. Both individuals of a twin pair share the same maternal environment and, in case of monozygotic twins, share the same fetal genes. Each fetus has its own fetoplacental environment, which may differ substantially from that of its co-twin [14]. In the context of fetal programming on cognitive outcomes, we studied placental mtDNA content at birth and intelligence in childhood in a study conducted in twins. Furthermore, we examined the association between placental mtDNA content and child problem behaviour. We hypothesize that placental mtDNA content at birth is associated with intelligence in childhood. In addition, we investigate if intra-pair differences in mtDNA content are associated with intra-pair differences in intelligence.

\section{Materials and methods}

\section{Subject recruitment}

The East Flanders Prospective Twin Survey (EFPTS) is a population based register of multiple births in the province of East-Flanders (Belgium) since 1964 [15]. 376 twin pairs born between 1982 and 1992 were invited, in 1992 and between 1996 and 1999 child IQ was assessed with use of the WISC-R (Wechsler Intelligence Scale for Children-Revised) test [16, 17]. The parents filled out the Child Behaviour Checklist (CBCL) [16]. Of the 376 twin pairs with IQ assessment, we selected those $(\mathrm{n}=405$ individuals) of which we had placental samples and information about residential history. We excluded 30 participants from our analysis because DNA quality or concentration was insufficient $(n=4)$, because triplicate measurements of mtDNA content were too variable (difference in quantification cycle more than 0.30) $(n=18)$ or missing data $(n=8)$. The number of children included in our analysis was 375 . Informed consent was obtained from all participants, and ethical approval was given by the Ethics Committee of University Hospital Ghent and Hasselt University (Registration number: B670201730788).

Data recorded by the obstetrician at birth included gestational age, birth weight and sex of the twins. Gestational age was calculated as the number of completed weeks of pregnancy and was estimated on the basis of last menstrual period combined with real-time ultrasonography in early pregnancy. At time of birth, placentas were examined within $24 \mathrm{~h}$ after delivery by a trained midwife following a standardized protocol [18]. Cord insertion was categorized into two groups: central insertion (central, paracentral, paramarginal) and peripheral insertion (marginal, membrane septum and membrane peripheral). For each individual, one placental biopsy was taken close to the surface near the insertion of the umbilical cord, also in twins sharing one placenta, and stored at $-20{ }^{\circ} \mathrm{C}$ in a biobank. Zygosity was determined by sequential analysis based on sex, choriontype, blood group determined on umbilical cord blood, placental alkaline phosphatase, and, since 1982, DNA fingerprints [19]. After DNA-fingerprinting, a zygosity probability of $99.9 \%$ was reached.

Further we collected information on parental education. Educational level as a proxy of socioeconomic status was categorized into three groups according to the Belgian education system: [1] no education or primary school, [2] lower secondary education and [3] higher secondary education or tertiary education.

Residential addresses of the mothers at birth were geocoded. Urban areas in a $5000 \mathrm{~m}$ radius from address was estimated based on Corine land cover 1990 (European Environment Agency). In addition to the individual levels of social economic class indicators, we gathered information on neighbourhood socioeconomic status. Based on their home address, all mothers were assigned to statistical sectors (average area $=1.55 \mathrm{~km}^{2}$ ), the smallest administrative entity for which statistical data are produced by the Belgian National Institute of Statistics (NIS). Belgian census data (FOD Economie/DG Statistiek) derived from the NIS were used to define neighbourhood household income based on annual household income in the year 1994.

\section{Assessment of neurodevelopment and behaviour during childhood}

The neurodevelopment outcome was assessed by the Wechsler Intelligence Scale for Children-Revised (WISC$\mathrm{R})$. The WISC-R consists of six verbal and six performance subscales and has been validated for use in this population [20]. The verbal subscales are Information (INF), Similarities (SIM), Arithmetic (ARI), Vocabulary (VOC), Comprehension (COM) and Digit Span (DS). The performance subscales are Picture Completion (PC), Picture Arrangement (PA), Block Design (BD), Object Assembly (OA), Coding (COD) and Mazes (MAZ). The scores on the subscales are standardized for age and added up to Verbal (VIQ), Performance (PIQ) and Total Intelligence Quotients (TIQ). In this study, the total 
scores of the subscales and the TIQ score were analysed. The minimum and maximum scores that can be obtained for total, verbal and performance IQ are 50 and 150 points.

Neurobehaviour was assessed by the Achenbach Child Behaviour Checklist (CBCL). This checklist was developed by Achenbach (1991) to examine the extent to which children have behavioural and emotional problem as perceived by their parents [21]. Although the CBCL allows for the calculation of separate scores corresponding to several behavioural dimensions, in this study we examined the total problem score, and the externalizing and internalizing subdomains.

\section{Measurement of mtDNA content}

DNA was isolated from placental tissue using the QIAamp DNeasy blood and tissue kit (Qiagen, Venlo, The Netherlands). Quality and concentration of the isolated placental DNA was assessed using the Nanodrop 1000 spectrophotometer (Isogen Life Science, Belgium) and the Quant-iT ${ }^{\mathrm{TM}}$ PicoGreen dsDNA Assay Kit (Life Technologies, United States) using the Omega Fluostar plate reader.

The method for measuring mtDNA content was described previously $[10,22]$. Relative mtDNA content was measured in placental tissue using a quantitative real-time polymerase chain reaction (qPCR) assay by determining the ratio of two mitochondrial gene copy numbers MTF3212/R3319 and MT-ND1 to a singlecopy nuclear control gene RPLPO. Reaction mixture and PCR cycles used are given in Additional file 1. Each PCR reaction was carried out in triplicate and three non-template controls as well as six inter-run calibrators were included on each 384-well plate. All samples were analyzed with the 7900HT Fast Real-Time PCR system (Life Technologies).

After thermal cycling, raw data was collected and processed. $\mathrm{C}_{\mathrm{T}}$ (cycle threshold)-values of the two mitochondrial genes were normalized relative to a nuclear reference gene according to the qBase software (Biogazelle, Zwijnaarde, Belgium). The program uses modified software based on the classic comparative $C_{T}$ method, it takes the reference gene into account and uses interrun calibration algorithms to correct for run-to-run differences [23]. The coefficient of variation for the mtDNA content in inter-run samples was $5.1 \%$. All samples were analyzed in triplicate and included in the study when the difference in quantification cycle $(\mathrm{Cq})$ value was $<0.30$.

\section{Statistical analysis}

For data management and statistical analyses, we used SAS software, version 9.4 (SAS Institute, Cary, NC). All reported $\mathrm{p}$ values are two-sided and were considered statistically significant when $\mathrm{p}<0.05$. The distribution of all variables was inspected. To improve normal distribution, mtDNA content was $\log _{10}$-transformed. Mixed modelling was performed to investigate intelligence in association with placental mtDNA content. The twins were analyzed as individuals in a multilevel regression analysis to account for relatedness between twin members by adding a random intercept to the model. The variance-covariance structure was allowed to differ between the three zygosity and chorionicity groups. Mixed modelling was performed adjusted for covariates selected a priori, namely sex, birth weight, gestational age, birth year, zygosity and chorionicity, cord insertion, age at measurement, parental educational level, neighbourhood household income, maternal smoking during pregnancy and urban environment. Mixed models were also used to study the association between child behaviour and placental mtDNA content, adjusting for previous mentioned covariates.

Subsequently, intra-pair differences in mtDNA content were calculated. These intra-pair differences were correlated with the intra-pair difference in total, verbal and performance IQ. Furthermore, the probability of the twin with the highest mtDNA content having the highest IQ in comparison with their co-twin was investigated using a Chi-square test. The analysis was stratified for zygosity and chorionicity, resulting in three groups; dizygotic, monozygotic-dichorionic and monozygotic-monochorionic twin pairs.

\section{Results}

\section{Characteristics of the study population}

Table 1 summarizes the characteristics of the study population including 201 mothers and the 375 children: $93.0 \%$ $(\mathrm{n}=348)$ of the participants included both twins from each twin pair, whereas the remaining $7.0 \%(n=27)$ only had one participating twin from each twin pair. Overall, most parents (56.7\%) were highly educated. Among the mothers, $8.0 \%(\mathrm{n}=16)$ continued smoking during pregnancy. The twin population comprises 194 (51.7\%) males and $181(48.3 \%)$ females. The twins had a mean $( \pm S D)$ birth weight of $2525 \pm 514 \mathrm{~g}$, an overall mean gestational age of 36.8 weeks (range 29-42) and 50\% was born preterm. Our analysis included 56.5\% dizygotic twins, 19.5\% monozygotic-dichorionic twins and $24.0 \%$ monozygotic-monochorionic twins. The mean $( \pm S D)$ relative placental mtDNA content was lower in the monozygoticmonochorionic twins $(0.98 \pm 0.47)$ compared with the dizygotic-dichorionic twins $(1.13 \pm 0.55, p=0.03)$ and monozygotic-dichorionic twins $(1.13 \pm 0.51, p=0.05)$. This is shown in Table 2. The twins completed the Wechsler Intelligence Scale for Children-Revised test at a mean age of 11.5 years old (between the ages of 8 and 
Table 1 Study population characteristics

\begin{tabular}{|c|c|}
\hline Parental characteristics & $(n=201)$ \\
\hline \multicolumn{2}{|l|}{ Education } \\
\hline Low & $16(8.0)$ \\
\hline Middle & $71(35.3)$ \\
\hline High & $114(56.7)$ \\
\hline Neighbourhood income, euro & $18,483 \pm 2474$ \\
\hline Smoking during pregnancy & $16(8.0)$ \\
\hline Cigarettes/day during pregnancy & $1.5 \pm 3.7$ \\
\hline Urban environment within $5 \mathrm{~km}$, \% & $32 \pm 12$ \\
\hline Characteristics of twins & $(n=375)$ \\
\hline \multicolumn{2}{|l|}{ Birth } \\
\hline Birth weight (g) & $2525 \pm 514$ \\
\hline Gestational age, weeks & $36.8 \pm 2.3$ \\
\hline Birth year & $1986 \pm 2.5$ \\
\hline \multicolumn{2}{|l|}{ Sex } \\
\hline Male-male & $143(38.1)$ \\
\hline Female-female & $131(34.9)$ \\
\hline Male-female & $101(26.9)$ \\
\hline \multicolumn{2}{|l|}{ Zygosity-chorionicity } \\
\hline Dizygotic-dichorionic & $212(56.5)$ \\
\hline Monozygotic-dichorionic & $73(19.5)$ \\
\hline Monozygotic-monochorionic & $90(24.0)$ \\
\hline \multicolumn{2}{|l|}{ Twins in final study } \\
\hline Twin with 1 individual in study & $27(7.0)$ \\
\hline Twin with 2 individuals in study & $348(93.0)$ \\
\hline \multicolumn{2}{|l|}{ Childhood } \\
\hline Age & $11.5 \pm 1.6$ \\
\hline \multicolumn{2}{|l|}{ Intelligence score (IQ) } \\
\hline Total IQ & $106.0 \pm 15.1$ \\
\hline Verbal & $106.6 \pm 14.6$ \\
\hline Performance & $103.9 \pm 15.3$ \\
\hline
\end{tabular}

Data presented are mean \pm standard deviation or number (percentage)

Table 2 Relative placental mtDNA content according to the different zygosity-chorionicity groups

\begin{tabular}{llll}
\hline $\begin{array}{l}\text { Relative placental mtDNA } \\
\text { content }\end{array}$ & Mean & $\begin{array}{l}\text { Standard } \\
\text { deviation }\end{array}$ & $\begin{array}{l}\text { p-value } \\
\text { (compared } \\
\text { to MZMC) }\end{array}$ \\
\hline Dizygotic-dichorionic & 1.13 & 0.55 & 0.03 \\
Monozygotic-dichorionic & 1.13 & 0.51 & 0.05 \\
Monozygotic-monochorionic & 0.98 & 0.47 & - \\
\hline
\end{tabular}

15 years). Their averaged scores $( \pm \mathrm{SD})$ were $106.0 \pm 15.1$ for total IQ, $106.6 \pm 14.6$ for verbal IQ and $103.9 \pm 15.3$ for the performance IQ.

\section{Determinants of childhood intelligence}

Table 3 shows the covariates associated with the total, verbal and performance intelligence quotient. The multivariable model is a mixed model taking the relatedness between twin member into account and is adjusting for sex, birth weight, gestational age, birth year, zygosity and chorionicity, cord insertion, age at measurement, parental educational level, neighbourhood household income, maternal smoking during pregnancy and urban environment. Total, verbal and performance IQ were higher in descendants from higher educated parents, whereas these IQ indexes were inversely associated with birth year of participants.

\section{Placental mtDNA content in association with intelligence}

Placental mtDNA content is associated with childhood intelligence 8 to 15 years later. Each doubling in mtDNA content is associated with a 2.0 point $(95 \%$ CI 0.02 to 3.9; $\mathrm{p}=0.05$ ) higher in total IQ (Table 4). This association is driven by performance IQ. A doubling in placental mtDNA content is associated with a 2.3 point $(95 \%$ CI 0.2 to $4.4 ; \mathrm{p}=0.03$ ) higher performance IQ while we observed no association with verbal IQ. The association between placental mtDNA content and performance IQ remains significant when additional adjusting for behaviour problems (total CBCL $\mathrm{T}$ score). This is shown in Additional file 1: Table S2.

To unravel the complex interplay between early-life environmental and genetic risk factors and to improve the causal interpretation of our study we made use of the specific twin design. Pearson correlations between intra-pair differences in mtDNA content and intelligence quotient stratified by twin zygosity-chorionicity are shown in Fig. 1. Significant associations were observed in monozygotic-monochorionic twin pairs $(\mathrm{n}=38)$ between placental mtDNA content and total $(\mathrm{r}=0.41, \mathrm{p}=0.01)$, verbal IQ $(r=0.42, p=0.009)$ and performance IQ $(\mathrm{r}=0.32, \mathrm{p}=0.05)$. These results in monozygotic-monochorionic twins show further that the twin with the highest placental mtDNA content at birth is 1.9 times more likely $(p=0.05)$ to have the highest total IQ of the twins. This was not observed in dizygotic-dichorionic twin pairs $(\mathrm{n}=102)$, neither in monozygotic-dichorionic twin pairs $(\mathrm{n}=34)$.

\section{Placental mtDNA content and behaviour problems}

No significant associations were observed between placental mtDNA content and total behaviour problems, neither for externalizing and internalizing problems (Table 5).

\section{Discussion}

Adaptive responses of the placenta may influence central nervous system development [1-3]. The key finding of our study was that placental mtDNA content is associated with child and adolescent IQ. Until now only studies 
Table 3 Covariates in association with a change in intelligence quotient

\begin{tabular}{|c|c|c|c|}
\hline \multirow[t]{2}{*}{ Covariates } & \multicolumn{3}{|c|}{ Multi variable model } \\
\hline & Change in IQ & $95 \% \mathrm{Cl}$ & $p$-value \\
\hline \multicolumn{4}{|l|}{ Covariates total intelligence quotient } \\
\hline Girls & -2.57 & -5.32 to 0.17 & 0.07 \\
\hline Birth weight, + IQR & 1.81 & -0.36 to 3.98 & 0.10 \\
\hline Gestational age, + IQR & 0.17 & -1.67 to 2.00 & 0.86 \\
\hline Birth year, +1 year & -1.99 & -3.03 to -0.96 & 0.0002 \\
\hline Peripheral cord insertion & 1.31 & -1.39 to 4.00 & 0.34 \\
\hline Age at measurement, +1 year & -1.50 & -3.06 to 0.06 & 0.06 \\
\hline High parental educational level & 6.99 & 4.19 to 9.79 & $<0.0001$ \\
\hline Neigbourhood household income, + IQR & -0.42 & -2.10 to 1.25 & 0.62 \\
\hline Maternal smoking during pregnancy & -1.46 & -6.21 to 3.29 & 0.55 \\
\hline Urban environment, + IQR & 2.40 & -0.03 to 4.83 & 0.05 \\
\hline \multicolumn{4}{|l|}{ Covariates verbal intelligence quotient } \\
\hline Girls & -3.52 & -6.24 to -0.79 & 0.01 \\
\hline Birth weight, + IQR & 1.78 & -0.37 to 3.94 & 0.11 \\
\hline Gestational age, + IQR & -0.13 & -1.88 to 1.62 & 0.88 \\
\hline Birth year, +1 year & -2.21 & -3.15 to -1.26 & $<0.0001$ \\
\hline Peripheral cord insertion & 0.87 & -1.79 to 3.54 & 0.52 \\
\hline Age at measurement, +1 year & -1.83 & -3.26 to -0.40 & 0.01 \\
\hline High parental educational level & 6.76 & 4.14 to 9.38 & $<0.0001$ \\
\hline Neigbourhood household income, + IQR & -0.98 & -2.55 to 0.58 & 0.22 \\
\hline Maternal smoking during pregnancy & -2.29 & -6.73 to 2.14 & 0.31 \\
\hline Urban environment, + IQR & 1.54 & -0.77 to 3.84 & 0.19 \\
\hline \multicolumn{4}{|l|}{ Covariates performance intelligence quotient } \\
\hline Girls & -1.50 & -4.48 to 1.47 & 0.32 \\
\hline Birth weight, + IQR & 0.99 & -1.31 to 3.28 & 0.40 \\
\hline Gestational age, +IQR & 0.51 & -1.41 to 2.43 & 0.60 \\
\hline Birth year, +1 year & -1.18 & -2.27 to -0.09 & 0.03 \\
\hline Peripheral cord insertion & 0.56 & -2.28 to 3.39 & 0.70 \\
\hline Age at measurement, +1 year & -0.71 & -2.35 to 0.93 & 0.40 \\
\hline High parental educational level & 5.57 & 2.65 to 8.49 & 0.0003 \\
\hline Neigbourhood household income, + IQR & 0.14 & -1.61 to 1.88 & 0.88 \\
\hline Maternal smoking during pregnancy & -0.09 & -5.04 to 4.86 & 0.91 \\
\hline Urban environment, + IQR & 2.59 & 0.08 to 5.11 & 0.05 \\
\hline
\end{tabular}

$\mathrm{Cl}$ confidence interval, IQR interquartile range

Table 4 Estimated change in intelligence quotient (IQ) for a doubling in mitochondrial DNA content

\begin{tabular}{llrl}
\hline $\begin{array}{l}\text { Intelligence } \\
\text { quotient (IQ) }\end{array}$ & Change in IQ & $\mathbf{9 5 \% ~ C l}$ & $\boldsymbol{p}$-value \\
\hline Total & 1.98 & 0.02 to 3.93 & 0.05 \\
Verbal & 1.42 & -0.51 to 3.34 & 0.15 \\
Performance & 2.29 & 0.21 to 4.37 & 0.03
\end{tabular}

Adjusted for sex, gestational age, birth weight, birth year, zygosity and chorionicity, cord insertion, age at IQ measurement, indicators of socioeconomic status (parental education and neighbourhood household income), smoking during pregnancy and urban environment

$\mathrm{Cl}$ confidence interval in elderly noted an association between mtDNA content in blood and cognitive performance [11, 12]. This is the first study investigating placental mtDNA content and intelligence one decade later.

Our study benefits from its specific twin design to achieve a higher degree of causality from our observational data. Significant intra-pair differences between placental mtDNA content and intelligence were only observed in monozygotic-monochorionic twins. As monozygotic twins are genetically identical, the association between the intra-pair differences in mtDNA content and IQ must be due to the individual fetoplacental 


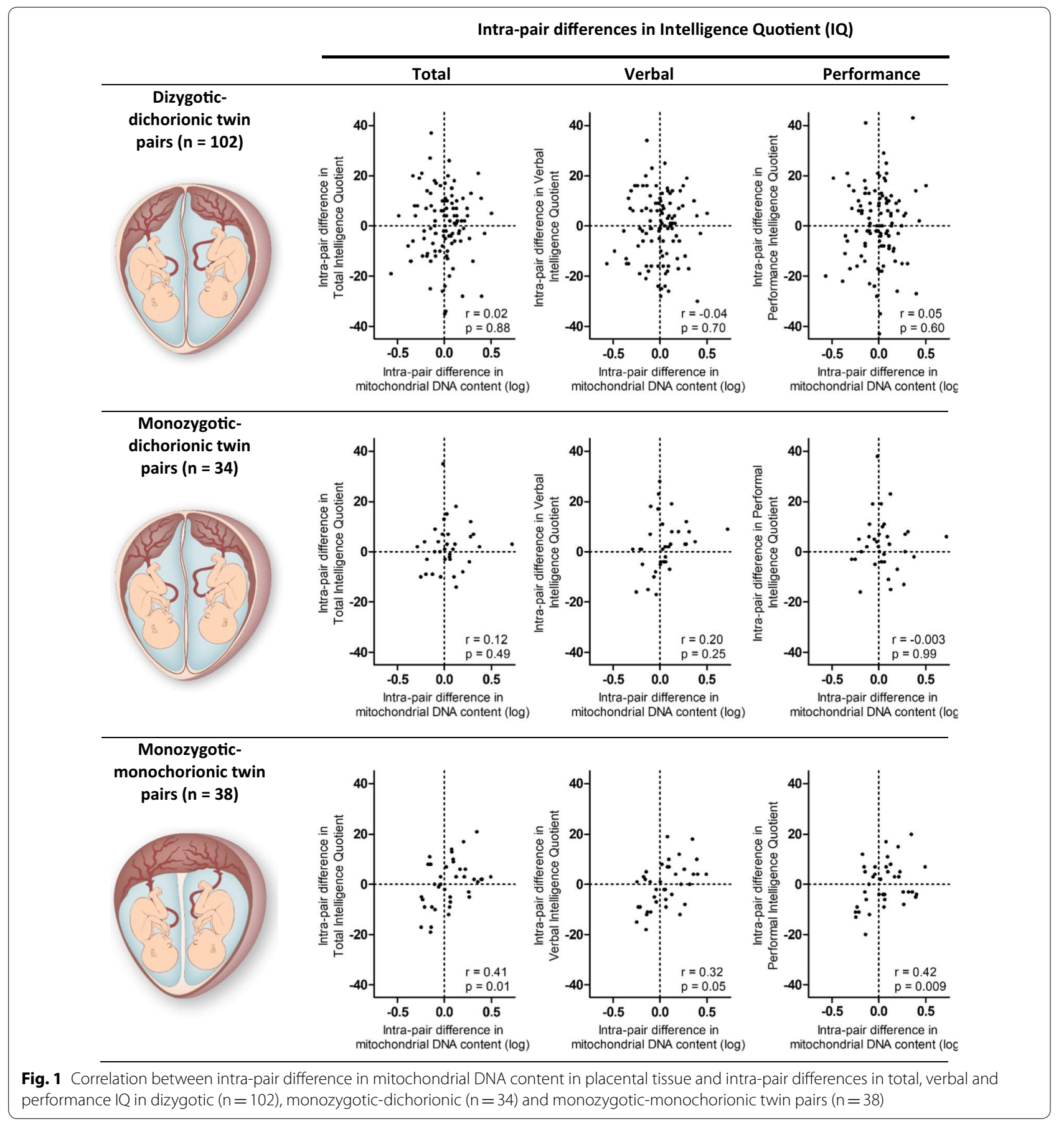

environment and specifically excludes the influence of genetic factors. Although monochorionic twins share only one placenta, intra-individual differences can be the result of an unequal degree of placental sharing [14]. In addition, placental sharing also enables differences between the individuals of the twin pair by allowing vascular connections between the circulation of the two fetuses and a higher prevalence of peripheral cord insertions [14]. In monozygotic-monochorionic twins unequal sharing of the placenta has been associated with a greater risk for birth weight discordance [24].

The significance of the fetal environment on intelligence in childhood is evident from twin studies, showing that twins score 4 point lower on an IQ test than singletons [25]. This difference is partially attributed to difference in intrauterine growth [25], nutrient deficiency, and 
Table 5 Estimated change in behaviour problems (CBCL T score) for a doubling in mitochondrial DNA content

\begin{tabular}{lccc}
\hline Behaviour problems & $\begin{array}{l}\text { Change } \\
\text { in CBCL T } \\
\text { score }\end{array}$ & $\mathbf{9 5 \% ~ C l}$ & $\boldsymbol{p}$-value \\
\hline Externalizing & 0.07 & -1.36 to 1.51 & 0.92 \\
Internalizing & -0.43 & -1.95 to 1.08 & 0.58 \\
Total & -0.60 & -2.02 to 0.81 & 0.40 \\
\hline
\end{tabular}

Adjusted for sex, gestational age, birth weight, birth year, zygosity and chorionicity, cord insertion, age at IQ measurement, indicators of socioeconomic status (parental education and neighbourhood household income), smoking during pregnancy and urban environment

$\mathrm{Cl}$ confidence interval

shorter gestational length of twins [26]. Several studies show associations between mtDNA content in placental tissue and life-style or environmental parameters at birth. In the Belgian ENVIRONAGE birth cohort, placental mtDNA content was $21.6 \%$ lower in mothers who smoked during pregnancy [27] and 17.4\% lower for each $10 \mu \mathrm{g} / \mathrm{m}^{3}$ increment in $\mathrm{PM}_{10}$ exposure during the third trimester of pregnancy [10]. In a Spanish birth cohort (INMA), a positive association was observed between placental mtDNA content and birth weight [28]. Moreover, mtDNA content was postulated as a potential mediator of the association between prenatal air pollution exposure and birth weight [28]. Until now, potential consequences of decreased mtDNA content in early life on outcomes later in life were unknown.

The exact pathways underlying the association between mtDNA content in placental tissue and intelligence in childhood remains unclear. It is known that placental mitochondria play an important role in functioning of the placenta. Therefore, we assume that an insufficient performance of the placenta, as a results of low mtDNA content, might affect the brain of the developing fetus or alternatively - as indicated in previous indicated in experimental work-that the placenta reflects similar molecular signatures as in the fetal brain [1-3]. Besides its role in nutrient transfer and gas exchange, the placenta plays a key role in transfer and synthesis of neuroactive factors that are necessary for normal brain development $[29,30]$. Neurotransmitter serotonin, essential for neurodevelopment, is not only provided by fetal and maternal sources but also synthetized by the placenta [31]. This is crucial for fetal brain development [1]. In addition, progesterone is synthesized by proteins located in the inner mitochondrial membrane of syncytiotrophoblast cells in the placenta [32]. Progesterone production by the placenta has a key role in providing precursors for the synthesis of neuroactive steroids in the fetal brain [33]. This highlights the importance of interactions between the placenta and the fetal brain [34]. Second, the most vulnerable early stages of fetal brain development depend on the placental transfer of maternal thyroid hormone [35]. Janssen et al. [27] observed that higher placental mtDNA content is associated with thyroid function more specifically with higher levels of free thyroid hormones in cord blood $\left(\mathrm{FT}_{3}, \mathrm{FT}_{4}\right)$. A decrease in placental mtDNA content could be the result of hormone changes influencing optimal functioning of the placenta. Indirectly this could affect the development of the fetusus and their early brain.

Our findings in newborns parallels those in adult populations. Indeed, a significant reduction in mtDNA content is observed in brain tissue of patients with neurodegenerative diseases, such as Parkinsons's and Alzheimer's disease $[36,37]$. The brain is susceptible to mitochondrial dysfunction due to its high energy demand [38] and because the brain, unlike most other tissues, depends exclusively on oxidative phosphorylation and glycolysis to create energy [39]. Furthermore neurotransmission depends on mitochondria not only for energy production but also for maintaining calcium homeostasis with the presynaptic terminal [40].

We observed a significant association between placental mtDNA content on performance IQ while no significant association was observed with verbal IQ. Verbal intelligence includes aspects like vocabulary and comprehension of language, while performance (nonverbal) intelligence includes matrix reasoning and picture completion. Our findings suggest that the regions of the brain associated with performance IQ are particularly affected by changes in placental mtDNA content during crucial periods of in utero development. Variations in placental function or other environmental insults during pregnancy such as maternal nutrition, might alter neurodevelopment in the fetus by promoting some brain regions over others [41].

The present study should be interpreted in the context of its limitations. First, an earlier study of Janssen et al. assessed within-placental variability of mtDNA content in a random subset of six placentas by comparing biopsies taken at four standardized sites across the middle region of the placenta [10]. mtDNA content within each placenta varied by a mean of $19.3 \%$ across the quadrants. To minimize the impact of within-placental variability in our study, biopsies used for mtDNA content assays were all taken at a fixed location. Second, it is a limitation that we have no information on cell composition of the placental biopsies. Third, we do not have information on vascular anastomoses for all twins. This may have an influence on placental sharing. Furthermore, our results show that placental mtDNA content is associated with IQ one decade later. Although its prospective nature, the present study is observational and cannot prove 
causation. However, the advantage of a twin study is that it can provide information on within-pair effects which increase causal interpretation. In this intra-pair analysis, we found in accordance with our primary analysis that the twin pair with the highest placental mtDNA content was 1.9 times more likely to have the highest score on total IQ 8 to 15 years later. Therefore, our study complies with important Hill criteria to establish causality. As our study is the first linking placental mitochondrial DNA content with childhood IQ others studies should confirm our association, preferentially also in a non-twin population to show the generalizability of the results.

For each doubling in placental mtDNA content we noted a 2.0 points increase in total intelligence and a 2.3 increase in performance IQ in childhood. Assuming causality, the magnitude of our estimates show that our molecular findings are relevant in a public health context. Indeed, although the impact on the population mean on the IQ might be moderate, children with a low placental mtDNA content will gravitate to the lower tail of the normal curve. Therefore, factors (both genetic, epigenetic and environmental) resulting in a lower placental mtDNA content might cause an inevitable "shift" in populations IQ. This has been demonstrated by Needleman for lead exposure during childhood, a greater proportion of children will gravitate to the lower tail of the normal curve $[42,43]$.

\section{Conclusions}

Our study is the first to show an association between placental mitochondrial function as exemplified by placental mtDNA content and cognitive outcomes in 8-15 year olds. Our results emphasize the importance of the intrauterine environment on intelligence and the role of placental mitochondrial functions.

\section{Supplementary information}

Supplementary information accompanies this paper at https://doi. org/10.1186/s12967-019-2105-y.

Additional file 1: Text S1. Mitochondrial and single copy-gene reaction mixture and PCR cycling conditions. Table S1. Primer sequences. Table S2. Estimated change in intelligence quotient (IQ) for a doubling in mitochondrial DNA content.

\section{Abbreviations}

CBCL: Child Behaviour Checklist; Cq: quantification cycle; $\mathrm{C}_{\mathrm{T}}$ : cycle threshold; DOHaD: The Developmental Origins of Health and Disease; EFPTS: The East Flanders Prospective Twin Survey; IQ: intelligence quotient; IQR: interquartile range; MT-ND1: mitochondrial encoded NADH dehydrogenase 1; mtDNA: mitochondrial DNA; MTF3212/R3319: mitochondrial forward primer from nucleotide 3212 and reverse primer from nucleotide 3319; PIQ: performance intelligence quotient; PM: particulate matter; qPCR: quantitative real-time polymerase chain reaction; RPLPO: acidic ribosomal phosphoprotein PO; TIQ: total intelligence quotient; VIQ: verbal intelligence quotient; WISC-R: Wechsler Intelligence Scale for Children-Revised.
Acknowledgements

Not applicable.

\section{Authors' contribution}

TSN, CD, ET, SW and EMB designed the study; CD, ET, EMB did data collection; $B G J$ optimized the protocol to measure mtDNA content; EMB performed the mtDNA measurements in the placental samples; TSN and EMB analyzed the data; TSN and EMB wrote the first draft of the paper. All authors critically revised the manuscript. All authors read and approved the final manuscript.

\section{Funding}

Dr. Bijnens holds a fellow-ship from the Marguerite-Marie Delacroix foundation. Bram Janssen is a postdoctoral fellow of the Research Foundation Flanders (FWO). Mitochondrial DNA measurements were covered by a grant of FWO (G073315N). Since its start, the East Flanders Prospective Twin Survey has been partly supported by grants from the Fund of Scientific Research

Flanders and Twins, a non-profit Association for Scientific Research in Multiple Births (Belgium). The funding source had no involvement in any activities related to study design, collection, analysis and interpretation of data presented in this manuscript, writing of the report or in the decision to submit the article for publication.

\section{Availability of data and materials}

The datasets used and/or analysed during the current study are available from the corresponding author on reasonable request.

\section{Ethics approval and consent to participate}

Informed consent was obtained from all participants, and ethical approval was given by the Ethics Committee of University Hospital Ghent and Hasselt University (Registration number: B670201730788).

\section{Consent for publication}

Not applicable.

\section{Competing interests}

The authors declare that they have no competing interests.

\section{Author details}

${ }^{1}$ Centre for Environmental Sciences, Hasselt University, Agoralaan Building D, 3590 Diepenbeek, Belgium. ${ }^{2}$ Department of Obstetrics and Gynaecology, Ghent University Hospital, Corneel Heymanslaan 10, 9000 Ghent, Belgium. ${ }^{3}$ Centre of Human Genetics, University Hospitals Leuven, Herestraat 49, 3000 Leuven, Belgium. ${ }^{4}$ Department of Neurology, Ghent University Hospital, Corneel Heymanslaan 10, 9000 Ghent, Belgium. ${ }^{5}$ Department of Public Health \& Primary Care, Leuven University, Kapucijnenvoer 35, 3000 Leuven, Belgium.

Received: 8 May 2019 Accepted: 19 October 2019

Published online: 08 November 2019

\section{References}

1. Bonnin A, Levitt P. Fetal, maternal, and placental sources of serotonin and new implications for developmental programming of the brain. Neuroscience. 2011:197:1-7.

2. Broad KD, Keverne EB. Placental protection of the fetal brain during shortterm food deprivation. Proc Natl Acad Sci USA. 2011;108(37):15237-41.

3. Saenen ND, Plusquin M, Bijnens E, Janssen BG, Gyselaers W, Cox B, et al. In utero fine particle air pollution and placental expression of genes in the brain-derived neurotrophic factor signaling pathway: an ENVIRONAGE Birth Cohort Study. Environ Health Perspect. 2015;123(8):834-40.

4. Petanjek Z, Judas M, Kostovic I, Uylings HB. Lifespan alterations of basal dendritic trees of pyramidal neurons in the human prefrontal cortex: a layer-specific pattern. Cereb Cortex. 2008;18(4):915-29.

5. Petanjek Z, Kostovic I. Epigenetic regulation of fetal brain development and neurocognitive outcome. Proc Natl Acad Sci USA. 2012;109(28):11062-3.

6. Vos M, Lauwers E, Verstreken P. Synaptic mitochondria in synaptic transmission and organization of vesicle pools in health and disease. Front Synaptic Neurosci. 2010;2:139. 
7. Picard M, McEwen BS. Mitochondria impact brain function and cognition. Proc Natl Acad Sci USA. 2014;111(1):7-8.

8. Jeng JY, Yeh TS, Lee JW, Lin SH, Fong TH, Hsieh RH. Maintenance of mitochondrial DNA copy number and expression are essential for preservation of mitochondrial function and cell growth. J Cell Biochem. 2008;103(2):347-57.

9. Gemma C, Sookoian S, Alvarinas J, Garcia SI, Quintana L, Kanevsky D, et al. Mitochondrial DNA depletion in small- and large-for-gestational-age newborns. Obesity. 2006;14(12):2193-9.

10. Janssen BG, Munters E, Pieters N, Smeets K, Cox B, Cuypers A, et al. Placental mitochondrial DNA content and particulate air pollution during in utero life. Environ Health Perspect. 2012;120(9):1346-52.

11. Mengel-From J, Thinggaard M, Dalgard C, Kyvik KO, Christensen K, Christiansen L. Mitochondrial DNA copy number in peripheral blood cells declines with age and is associated with general health among elderly. Hum Genet. 2014;133(9):1149-59.

12. Lee JW, Park KD, Im JA, Kim MY, Lee DC. Mitochondrial DNA copy number in peripheral blood is associated with cognitive function in apparently healthy elderly women. Clin Chim Acta. 2010;411(7-8):592-6.

13. de Boo HA, Harding JE. The developmental origins of adult disease (Barker) hypothesis. Aust N Z J Obstet Gynaecol. 2006;46(1):4-14.

14. Loos RJ, Ridgway CL, Ong K. Theoretical underpinning of the use of twin studies in life course epidemiology. Family matters: designing, analysing and understanding family based studies in life course epidemiology. Oxford: Oxford University Press; 2009. p. 57-84.

15. Derom C, Thiery E, Peeters H, Vlietinck R, Defoort P, Frijns JP. The East Flanders Prospective Twin Survey (EFPTS): an actual perception. Twin Res Hum Genet. 2013;16(1):58-63.

16. Jacobs N, Rijsdijk F, Derom C, Danckaerts M, Thiery E, Derom R, et al. Child psychopathology and lower cognitive ability: a general population twin study of the causes of association. Mol Psychiatry. 2002;7(4):368-74.

17. Antoniou EE, Fowler T, Thiery E, Southwood TR, van Gestel S, Jacobs N, et al. Intrauterine environment and cognitive development in young twins. J Dev Orig Health Dis. 2013;4(6):513-21.

18. Derom R, Derom C, Vlietinck R. Placentation. In: Keith L, Papiernik E, Keith D, Luke B, editors. Multiple pregnancy: epidemiology, gestation \& perinatal outcome. New York: The Parthenon Publishing Group; 1995. p. 113-28.

19. Vlietinck R. Determination of the zygosity of twins. Leuven; 1986.

20. Wechsler D. Wechsler Intelligence Scale for Children-R (Dutch version): Swets and Zeitlinger B. V., Lisse, the Netherlands; 1986.

21. Achenbach T. Integrative guide for the 1991 CBCL/4-18, YSR, and TRF profiles. Burlington: Department of Psychiatry, University of Vermont; 1991

22. Pieters N, Koppen G, Smeets K, Napierska D, Plusquin M, De Prins S, et al. Decreased mitochondrial DNA content in association with exposure to polycyclic aromatic hydrocarbons in house dust during wintertime: from a population enquiry to cell culture. PLoS ONE. 2013;8(5):e63208.

23. Hellemans J, Mortier G, De Paepe A, Speleman F, Vandesompele J. qBase relative quantification framework and software for management and automated analysis of real-time quantitative PCR data. Genome Biol. 2007:8(2):R19.

24. Fick AL, Feldstein VA, Norton ME, Wassel Fyr C, Caughey AB, Machin GA. Unequal placental sharing and birth weight discordance in monochorionic diamniotic twins. Am J Obstet Gynecol. 2006;195(1):178-83.

25. Voracek $M$, Haubner T. Twin-singleton differences in intelligence: a metaanalysis. Psychol Rep. 2008;102(3):951-62.
26. Eriksen W, Sundet JM, Tambs K. Twin-singleton differences in intelligence: a register-based birth cohort study of Norwegian males. Twin Res Hum Genet. 2012;15(5):649-55.

27. Janssen BG, Gyselaers W, Byun HM, Roels HA, Cuypers A, Baccarelli AA, et al. Placental mitochondrial DNA and CYP1A1 gene methylation as molecular signatures for tobacco smoke exposure in pregnant women and the relevance for birth weight. J Transl Med. 2017;15(1):5.

28. Clemente DB, Casas M, Vilahur N, Begiristain H, Bustamante M, Carsin AE, et al. Prenatal ambient air pollution, placental mitochondrial DNA content, and birth weight in the INMA (Spain) and ENVIRONAGE (Belgium) birth cohorts. Environ Health Perspect. 2015;124(5):659-65.

29. Hirst JJ, Walker DW, Yawno T, Palliser HK. Stress in pregnancy: a role for neuroactive steroids in protecting the fetal and neonatal brain. Dev Neurosci. 2009;31(5):363-77.

30. Petraglia F, Imperatore A, Challis JR. Neuroendocrine mechanisms in pregnancy and parturition. Endocr Rev. 2010;31(6):783-816.

31. Whitaker-Azmitia PM. Serotonin and brain development: role in human developmental diseases. Brain Res Bull. 2001;56(5):479-85.

32. Martinez F, Olvera-Sanchez S, Esparza-Perusquia M, Gomez-Chang E, Flores-Herrera O. Multiple functions of syncytiotrophoblast mitochondria. Steroids. 2015;103:11-22.

33. Nguyen PN, Billiards SS, Walker DW, Hirst JJ. Changes in 5alpha-pregnane steroids and neurosteroidogenic enzyme expression in the perinatal sheep. Pediatr Res. 2003;53(6):956-64.

34. Hirst JJ, Kelleher MA, Walker DW, Palliser HK. Neuroactive steroids in pregnancy: key regulatory and protective roles in the foetal brain. J Steroid Biochem Mol Biol. 2014;139:144-53.

35. Korevaar TIM, Tiemeier H, Peeters RP. Clinical associations of maternal thyroid function with foetal brain development: epidemiological interpretation and overview of available evidence. Clin Endocrinol. 2018;89:129-38.

36. Pyle A, Anugrha H, Kurzawa-Akanbi M, Yarnall A, Burn D, Hudson G. Reduced mitochondrial DNA copy number is a biomarker of Parkinson's disease. Neurobiol Aging. 2016;38:216.e7-10.

37. Rice AC, Keeney PM, Algarzae NK, Ladd AC, Thomas RR, Bennett JP Jr. Mitochondrial DNA copy numbers in pyramidal neurons are decreased and mitochondrial biogenesis transcriptome signaling is disrupted in Alzheimer's disease hippocampi. J Alzheimers Dis. 2014;40(2):319-30.

38. Machado AK, Pan AY, da Silva TM, Duong A, Andreazza AC. Upstream pathways controlling mitochondrial function in major psychosis: a focus on bipolar disorder. Can J Psychiatry. 2016;61 (8):446-56.

39. Clay HB, Sillivan S, Konradi C. Mitochondrial dysfunction and pathology in bipolar disorder and schizophrenia. Int J Dev Neurosci. 2011:29(3):311-24.

40. Jonas E. BCL-xL regulates synaptic plasticity. Mol Interv. 2006;6(4):208-22.

41. Jones A, Osmond C, Godfrey KM, Phillips DI. Evidence for developmental programming of cerebral laterality in humans. PLOS ONE. 2011;6(2):e17071.

42. Needleman $\mathrm{HL}$. The persistent threat of lead: a singular opportunity. Am J Public Health. 1989;79(5):643-5.

43. Needleman HL, Leviton A, Bellinger D. Lead-associated intellectual deficit. N Engl J Med. 1982;306(6):367.

\section{Publisher's Note}

Springer Nature remains neutral with regard to jurisdictional claims in published maps and institutional affiliations. 